\title{
Characterization of Normal and Malignant Breast Tissues utilizing Hyperspectral Images and Associated Differential Spectrum Algorithm
}

\author{
Mohamed H. Aref ${ }^{1 *}$, Abou-Bakr M. Youssef ${ }^{2}$, Ibrahim H. Aboughaleb ${ }^{1}$, Yasser H. El-Sharkawy ${ }^{1}$ \\ ${ }^{1}$ Biomedical Engineering Department, Military Technical College, Cairo, Egypt \\ ${ }^{2}$ System \& Biomedical Engineering Department, Cairo University, Giza, Egypt \\ *e-mail: Mh-aref@ieee.org
}

\begin{abstract}
Breast malignancy is the most pervasive disease and a significant reason for death in women around the world. Recently, Photonic technologies play a vital role in medical applications. This study presents an outline of recent outcomes on the magnitude of breast tissue optical properties. We established an optical system setup utilizing a hyperspectral (HS) camera with poly-chromatic source lights with wavelength $(380 \sim 1050 \mathrm{~nm})$ for this investigation. Measuring the diffuse reflection (Rd) of the investigated ex vivo breast sample to select the optimum spectral image to differentiate between the normal and tumor in the near infra-red and visible (NIR-VIS) spectrum. Finally, applying the custom algorithm to increase the image contrast and applying contour delineation of the malignant regions. The experimental analysis indicates key spectroscopic variations between normal tissue and malignant region in range $(550 \sim 650 \mathrm{~nm})$. Although, after data normalization, there was noticeable variation at three ranges $(630-680 \mathrm{~nm}),(720-770 \mathrm{~nm})$, and $(830-880 \mathrm{~nm})$. The calculated standard deviation (S.d) between the normal and cancer tissue to validate the selective ranges shows that the highest contrast at wavelength $680 \mathrm{~nm}$. However, the histogram analysis illustrates that the spectral image at $600 \mathrm{~nm}$ was higher contrast and wavelength $400 \mathrm{~nm}$ was the lowest contrast from the select seven-spectral images $(400,500,600,700,800,900,1000 \mathrm{~nm})$ to avoid the processing time of the captured HS 128-frames. The proposed potential method could provide promising results on the investigated breast sample optical properties in the diagnostic applications to assist the pathologist and the surgeon. Where the optimum wavelength at $680 \mathrm{~nm}$ for diagnostic applications and the ideal spectral image at $600 \mathrm{~nm}$ discriminate between the normal and malignant tissue. (C) 2021 Journal of Biomedical Photonics \& Engineering.
\end{abstract}

Keywords: breast cancer early detection; hyperspectral imaging system; tissue optical properties; diffuse reflectance; spectral differences algorithm; optical spectroscopy.

Paper \#3384 received 30 Oct 2020; revised manuscript received 7 Apr 2021; accepted for publication 12 Apr 2021; published online 6 May 2021. doi: 10.18287/JBPE21.07.020302.

\section{Introduction}

Breast malignancy is the most predominant disease and a significant reason for death in women overall. Early determination is vital for expanding the survival rate among women with breast malignant growth [1-3]. In male patients, the most leading reason of cancer death is lung cancer. However, in females, breast cancer is mostly the leading cause of mortality [4-6]. More than $8 \%$ of women will endure this infection during their lifetime [7]. Breast cancer is a malignant tumor that is 
established from cells in the breast with no symptoms in the early stages [8].

Breast-conserving medical procedure stays challenging because of the absence of clinically accessible intraoperative resection edge strategies. Consequently, in up to $37 \%$ of women going through a breast-conserving medical procedure, the tumor is found in the resection edge of the resected sample [9-12]. This is a sign of tumor residual kept unintended inside the patient, which spreads the hazards for growing the tumor recurrence and increasing the reheeling time [13]. Consequently, these patients regularly require extra treatment like radiotherapy support or a reextraction [14]. Right now, a pathologist, who assesses the tissue under a magnifying lens, surveys the resection edge a couple of days after the medical procedure. In that capacity, no immediate input can be given to the specialist during the medical procedure $[12,15]$.

Conventional diagnosis techniques of histological samples depend on the manual assessment of morphological structures of samples by qualified pathologists. Recently, the utilization of computer-aided skills for improving these systems is a developing pattern to lessen the intra and inter-observer inconstancy [16]. Such innovations are expected to enhance the diagnosis, make it reproducible and quantitative, and reduce time in the assessment of the investigated samples [17, 18].

To decrease the quantity of tumor-positive resection edges, different methods for resection edge appraisal during the breast-conserving medical procedure have been proposed [19-21]. Currently, the available edge assessment methods are frozen section analysis, imprint cytology, specimen radiography, and ultrasound [7, 21-23]. Despite this, none of all these techniques could clinically extensive. With frozen section examination, tissue can be investigated in 30 min with a specificity and sensitivity of $95 \%$ and $83 \%$. Although, the main restrictions of this method are the necessity of a qualified pathologist, the possibility of false negatives (FN), and the feasibility of examining the entire resection surface [24, 25].

The hyperspectral imaging (HSI) system is a substitution modality for biological detection and RGB imaging for its ability to segregate between various materials by utilizing both morphological and spectral characteristics [26-28]. This tool has been effectively employed to support the diagnosis of various types of histological samples, for example, blood oxygenation and microcirculation [29-31], brain cancer detection [32, 32], tissue characterization [33-38], and monitoring liver ablation [39-44], and in the field of breast cancer $[15,45-48]$.

The female human being breast comprises principally glandular, fat, and connective tissue, along with blood and lymphatic vessels, and it contains a few basic mammary glands (lobes), each depleting through a different lactiferous duct [49-52]. Other than blood and lymphatic vessels, the connective tissue (stroma) comprises different stromal cells and the extracellular matrix (ECM) which comprises proteins, water, and polysaccharides [53]. The ECM addresses the platform for the stromal cells as well as passes on biochemical and biophysical signs to cells. breast malignancy development is related to changes in ECM arrangement, with inflammatory cell penetration, and separation of fibroblasts [54]. The spectral differences between the normal breast tissue and the biochemical changes of the malignant tissue which leading to these spectral features could be illustrated in Fig. A1 in the Appendix.

The red and near-infrared (NIR) light source wavelengths provide different propagation in the biological tissues regarding the other visible (VIS) spectrum. Where these sources could reach up to $10 \mathrm{~cm}$ thru the tissue and capable of cancer detection. Although, the NIR photons could freely path in the tissue till $\sim 10 \mathrm{~cm}$. However, the corresponding scattering is only 20 40 $\mu \mathrm{m}$ [48]. Additionally, the NIR imaging delivers a high affectability for molecular functions that are relatively near the surface. In any case, because of versatile scattering with different cellular components, NIR source light gets diffuse inside a couple of millimeters of engendering through tissue [55-57]. Finally, the main concern in this experiment was measuring the sample the diffuse reflection $\left(\mathrm{R}_{\mathrm{d}}\right)$ to identify and characterize the explored breast sample's optical properties regarding its spectral signature, as more clarified in Fig. 1.

In the presented prospective approach, we recognized a system that could offer promising results by investigating the breast sample optical properties for diagnostic applications purposes. Although, optics in the medical sector faced several fluctuations and development depending on the optical properties of the biological tissue. However, optical medical equipment contributes by presenting strategies and making of equipment utilized in the healthcare of different claims to specialties, such as oncology, general surgery, and ophthalmology. Finally, after exploring breast tissue optical properties diffuse reflection (Rd). We could select the optimum wavelength for breast tissue in diagnostic applications to assist the pathologist during the tissue investigation and reduce the time of examination and the surgeon during the breast biopsy and Mastectomy process.

\section{Materials and Methods}

This experimental investigation was a part of series of consecutive research studies in early breast cancer diagnostic. Where our main objective is to select the lowest and highest contrast spectral image to apply our custom subtraction algorithm and delineate the various tumor regions [45-47]. 




Fig. 1 The Light Interaction (Absorption / Diffuse Reflection / Reflectance) of ex vivo Breast Tissue Sample highlighting the Substitution of the breast Tissue Cells and its interaction with the various spectral range.

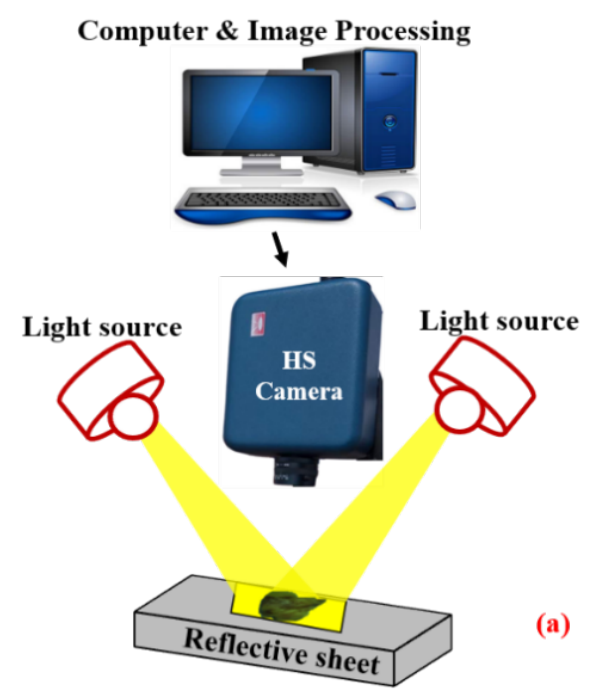



a)

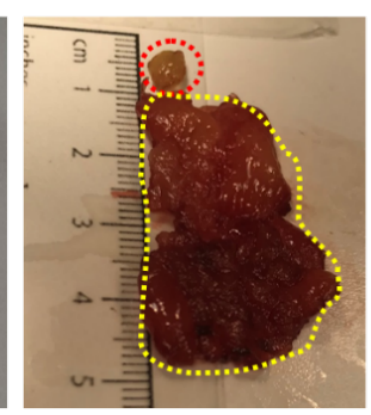

Malignant Tissue

Normal Tissue

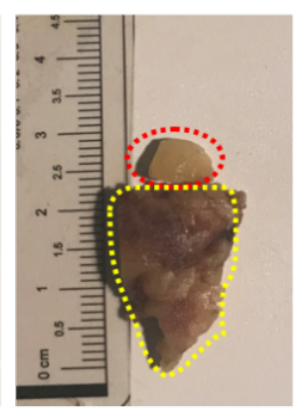

(b)

Fig. 2 (a) System Setup Measuring the Optical Properties of the Diffuse Reflection ( $\mathrm{R}_{\mathrm{d}}$ ) of the ex vivo Breast Sample; (b) Acquired RGB images for Some Examples of the ex vivo Breast Samples Exploited the Investigated Experiments.

\subsection{The main framework interconnections protocol could be explained as follows:}

$>$ Sample investigation \& Tissue characterization.

$>$ HS Image for the ex vivo breast sample.

$>$ The measurements for sample diffuse reflection $\left(\mathrm{R}_{\mathrm{d}}\right)$.
$>$ Selection of the spectral image to differentiate between Tumor and normal regions in the diagnostic applications.

$>$ Applying the custom algorithm to increase the image contrast (image enhancement) and delineation of the tumor regions. 
The main framework of the proposed system utilizing hyperspectral HS camera (Surface Optics, SOC710, USA) incorporated with polychromatic source light (Derungs, $150 \mathrm{~W}, 385 \sim 1050 \mathrm{~nm}$, Germany). Fig. 2(a) had been highlighting the schematic diagram for the exploited system to measure the investigated breast sample diffuse reflection $\left(\mathrm{R}_{\mathrm{d}}\right)$, to discriminate the optimum wavelength for differentiation between the normal and tumor regions in the investigated ex vivo breast sample by exploring the optical properties of spectroscopy in the Near and visible (NIR-VIS) spectrum.

\subsection{Pathology examination and sample slicing for malignant}

In advance of the experimental investigation, the procedure validation was achieved from "Ain Shams University - Faculty of Medicine - Ethics Committee". Ten patients who were encountering Breast cancer development experienced a total Mastectomy process. Subsequently, a careful examination with good evaluation, the breast tumor samples were selected from arbitrary patients, a cancer diagnosis was confirmed to the pathological report which is the ground truth for our investigation. All the patient and the investigated samples necessary data of the presented study was illustrated in Table A1 in the Appendix.

The investigated ex vivo breast samples were crudely cut into slices with approximate sizes $(2.5 \times 3 \mathrm{~cm})$, Sample thickness $4 \sim 6 \mathrm{~mm}$, then transported in icebox fill of deionized saline. The explored sample temperature $25 \sim 28{ }^{\circ} \mathrm{C}$ at lap temperature $23^{\circ} \mathrm{C} \sim 24{ }^{\circ} \mathrm{C}$. The samples persevered before and after the trails at $\left(-65 \sim-75^{\circ} \mathrm{C}\right)$ inside Lap Refrigerator (ThermoFisher Scientific, ULT2090, USA), as displayed in Fig. 2 (b).
The cancer regions were confirmed to the pathological report which is the ground truth for our investigation. Even though the pathologist could detect the cancerous breast tissues. However, this study aims to build an optical imaging system to assist the pathologist to differentiate between the normal or healthy tissue and the cancer tissue regions to reduce the time of the investigation and increasing the accuracy of the detection. Additionally, to support the surgeon in guidance during the tumor resection in real-time.

\subsection{The main theory and system equations}

The main block diagram of the system set up to measure the optical properties of the investigated breast samples, measuring the diffuse reflection $\left(\mathrm{R}_{d}\right)$ then applying the custom algorithm to increase the image contrast and delineate the tumor region of the samples, which is more identified in Fig. 3.

The transport theory is the main concept for light propagation in the tissue concerning the radiant power of the transferred light in the tissue surface $[58,59]$. Where, designating the light propagation in a turbid medium is vital to illustrate optical parameters, radiance, fluence proportion, and flux [34]. The photon scattering function with the power of photons, energy, velocity in the medium, as described in a previous study [45].

Often medical applications involve the interaction of light with the tissue. The amount of light could be expressed as the irradiance $\mathrm{E}_{0}$, which is realized as the radiant energy flux incident on an element of the surface, divided by the area of the surface. Apart from that incident light is reflected, and another entering the tissue is attenuated by scattering and absorption according to Beer's law, as highlighted in Eqs. (1) and (2).

System Block diagram for light diffuse reflection $\left(\mathbf{R}_{d}\right)$

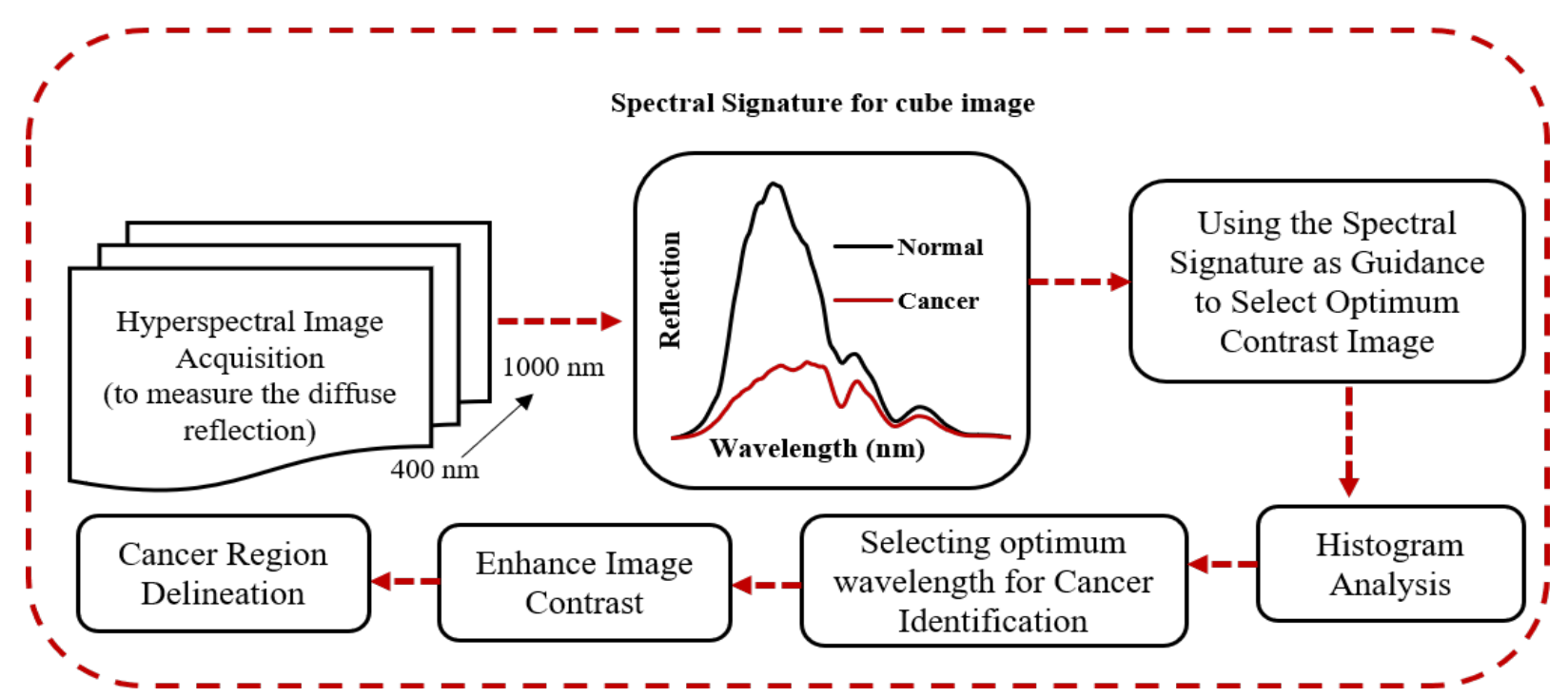

Fig. 3 The Image Processing Protocol for the System Setup to Measure the Optical Properties (Diffuse Reflection (R, )) for ex vivo Breast Tissue Sample. 


$$
\omega(\mathrm{d})={ }^{\prime} \mathrm{E}_{0}(1-\mathrm{R})\left(e^{\left(-\left(\mu_{a}-\mu_{s}\right) \mathrm{d}\right)}\right),
$$

where $\left(\mu_{s}\right)$ is the scattering attenuation, $\left(\mu_{a}\right)$ is the absorption attenuation, and $\omega(\mathrm{d})$ is the fluence rate for the un-scattered beam at position $\mathrm{d},{ }^{\prime} \mathrm{E}_{0}$ is the irradiance, and $\mathrm{R}$ is the Fresnel surface reflection.

$$
\sigma=\frac{1}{\left(\mu_{s}+\mu_{s}\right)}=\frac{1}{\mu_{t}}
$$

where $\left(\mu_{t}\right)$ is the total attenuation coefficient and $(\sigma)$ is the penetration depth

The light interaction of the light concerning the biological tissues is evaluated regarding the Transmission measurement (T), diffuse reflection $\left(R_{d}\right)$, and calculated attenuation absorption (A) [34], as presented in Eqs. (3) and (4).

$$
\begin{aligned}
& \mathrm{T}=\frac{\mathrm{T}_{s}-\mathrm{T}_{0}}{\mathrm{~T}_{s t}-\mathrm{T}_{0}} ; \\
& \mathrm{R}_{\mathrm{d}}=\frac{\mathrm{R} d_{s}-\mathrm{R} d_{0}}{\mathrm{R} d_{s t}-\mathrm{R} d_{0}},
\end{aligned}
$$

where the measured light intensities by diffuse reflectance/transmittance are $\left(\frac{\mathrm{R} d_{s}}{T_{s}}\right)$ and $\left(\frac{\mathrm{R} d_{s t}}{T_{s t}}\right)$ with the breast sample and the standard reflecting optical white plate respectively. The $\frac{R d_{0}}{T_{0}}$ demonstrates the captured background light intensity without a sample.

\subsection{Spectral planning and Procurement}

To capture the required HSI data, we utilized a Hyperspectral camera (Surface Optics, SOC710, USA) with a spectral resolution $4.68 \mathrm{~nm}$ corresponding to $600 \times 502$ pixels for the spectral cluster (the Cube image capturing time $=3.65 \mathrm{sec}$ ). The Hyperspectral camera covered overall 128 spectral clusters in range $379 \sim 1050 \mathrm{~nm}$. The camera is incorporated with a lens (Schneider, 400 1000 nm, Germany). The exploited source light for the Hyperspectral image scan, polychromatic source light (Derungs, $150 \mathrm{~W}$, $385 \sim 1050 \mathrm{~nm}$, Germany). The image processing exploits DADiSP / SE software (DSP Development Co., 6.7 B02, 2020, USA) on a personal Laptop (DELL, Intel Core i7, USA).

An essential step before capturing the investigated breast samples with the Hyperspectral camera, to capture a white (fully reflective) cube image and dark cube image (closing the camera cap) to remove the unnecessary artifacts and background noise [60], as more illustrated in Eq. (5):

$$
R F(\vartheta)=\frac{S i(\vartheta)-D i(\vartheta)}{W i(\vartheta)-D i(\vartheta)} \times 100 \%
$$

where $R F(\vartheta)$ is the relative diffuse reflectance of the sample image, $\operatorname{Si}(\vartheta)$ is the sample cube image, $\operatorname{Di}(\vartheta)$ is the dark cube image (closing the camera cap), and $W i(\vartheta)$ is the capture a white (fully reflective) cube image.
Even though to cover the spectral range (400 to $1000 \mathrm{~nm}$ ) we compute 128 frames with $4.68 \mathrm{~nm}$ resolution for each investigated breast sample. However, it is time-consuming and needs to make a lot of effort to analyze the 128 images, so from the one-dimensional (1D) signal of investigated breast samples, we optimize the wavelength ranges at which we can differentiate between the normal and the malignant to select the image around this wavelength.

Furthermore, to reduce the time of the image processing instead of the 128 frames, we examine the selection of the spectral image every $100 \mathrm{~nm}$ step, starting from $400 \mathrm{~nm}$ till $1000 \mathrm{~nm}$. Where, the selected spectral images were $(400,500,600,700,800,900$, $1000 \mathrm{~nm}$ ), so we can choose the closest result wavelength. Afterward, pertaining statistical analysis (histogram) to select the optimum highest contrast and lowest contrast image. Finally, applying the custom subtraction algorithm to remove the DC background noise, image enhancement, and apply the K-mean segmentation with contour delineation of the variable threshold regions of the tumor regions in the breast sample.

Next, applying the normalization on the selected image to remove the unwanted spectral impact from the polychromatic light. The captured original images various due to the light, the irregular shape of the sample, and temperature variations, spectral images ought to be normalized including pixel normalization, as demonstrated in a previous study [46]. Then, applying the Moving average filter, the arithmetic means filter at kernel value 10 for noise reduction and image enhancement [61], as clarified in Eq. (6):

$$
f(x \times y)=\frac{1}{q, t}+\sum_{(\mathrm{r} \times c) \in W}^{\infty} S(\hat{\mathrm{r}} \times \mathrm{c}),
$$

where $S$ is the noisy image, $f(x \times y)$ is the restored image, and $\mathrm{r}$ and $\mathrm{c}$ for the row and column coordinates respectively, within a window $\mathrm{W}$ of size $q \times t$ where the process takes place.

Finally, to select the optimum image to distinguish between the normal and malignant regions, we pertaining statistical analysis (histogram), which is corresponding to the bar graph to graphically represents vital information for the spectral over discrete intervals [62-64], as illustrated in Eq. (7):

$$
\mathcal{H}=\sum_{i=1}^{n} \mathcal{e} w_{i} \times f D_{i},
$$

where the Area of Histogram is $(\mathcal{H})$, the class width is $(\mathcal{C} w)$, and the frequency density is $(f \mathrm{D})$.

\section{Results}

In this section, we going to illustrate the fundamental investigation outcomes. The primary objective of these examinations is to highlight the system capability to discriminate between the normal and malignant tumors of investigated ex vivo breast samples utilizing the biological tissue's optical properties. 
We established an optical imaging system exploiting a hyperspectral (HS) camera with source light at wavelength range $(380 \sim 1050 \mathrm{~nm})$, for measurement of breast sample light diffuse reflectance $\left(\mathrm{R}_{\mathrm{d}}\right)$. To select the optimum wavelength which could be capable to differentiate between the normal and tumor precise regions in the ex vivo breast sample by exploring the optical properties spectroscopy in the near and visible (NIR-VIS) spectrum.

The raw data displayed in the graph of Fig. 4(a) signifies the typical spectral values of the measured diffuse reflectance $\left(\mathrm{R}_{\mathrm{d}}\right)$ from selected points on the malignant and normal regions. Where the discrimination between the malignant and normal regions regarding the intensity versus the wavelength. However, to avoid this problem, the raw data was normalized to neglect the variation of the intensity and focusing on the spectral change to display the spectroscopic point measurements, as demonstrated in Fig. 4(b).

The experiment average outcomes demonstrated that from the raw data signal in Fig. 4 (a) the lowest measured $\left(\mathrm{R}_{\mathrm{d}}\right)$ at wavelength $400 \mathrm{~nm}$ and after data normalization, there was noticeable variation at three ranges $(630-680 \mathrm{~nm}),(720-770 \mathrm{~nm})$, and $(830-880 \mathrm{~nm})$. The experiment was repeated on the whole Ten investigated samples to measure the $\left(\mathrm{R}_{\mathrm{d}}\right)$ of the normal tissue as displayed in the worksheet in Fig. A2 in the Appendix and the magnified image for the combination (Rd) signals of the normal tissue for the Investigated Eight-Samples regards the source light reference, as shown in Fig. A2(i) in the Appendix. Furthermore, the measured ( $\left.\mathrm{R}_{\mathrm{d}}\right)$ of the tumor tissue was presented in Fig. A3 in the Appendix and the magnified image for the combination $\left(\mathrm{R}_{\mathrm{d}}\right)$ signals of the tumor regions for the Investigated Eight-Samples regards the source light reference, as presented in Fig. A2(i) in the Appendix.
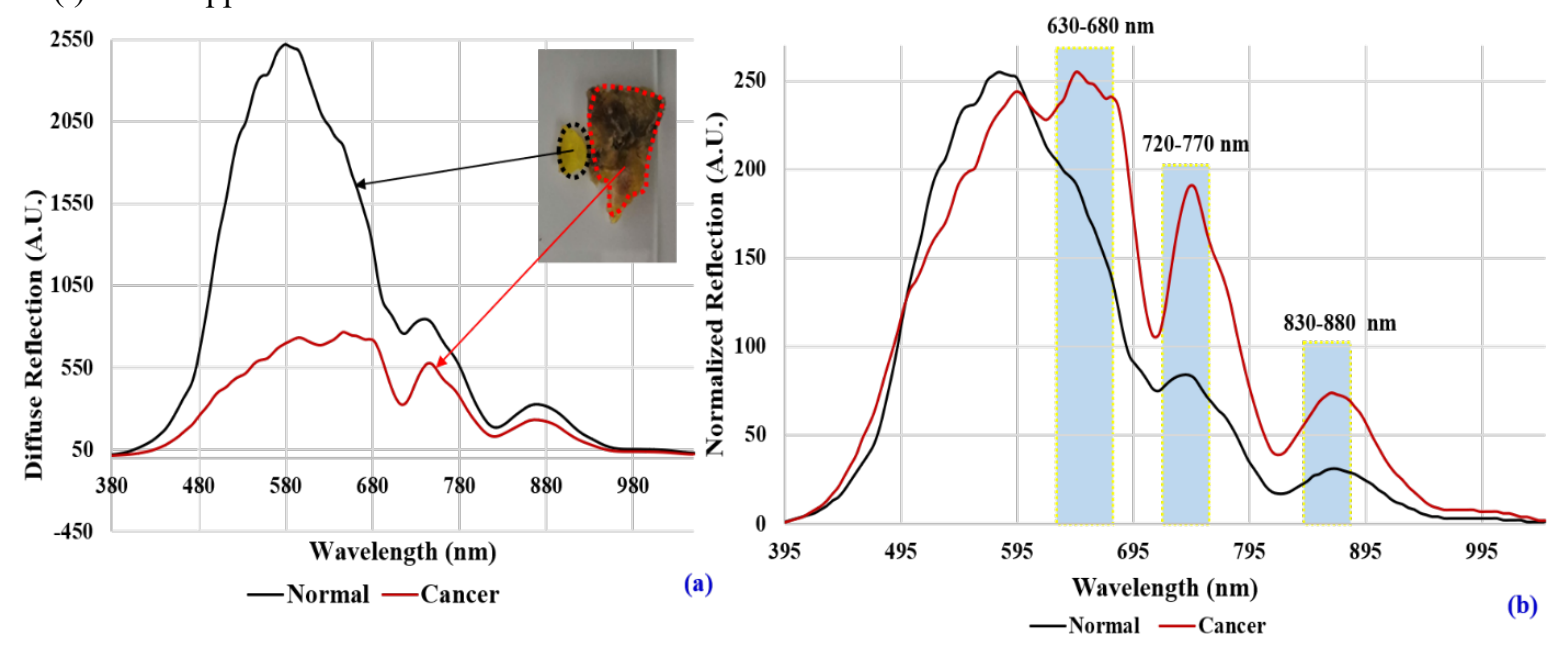

Fig. 4 (a) The raw data of the diffuse reflectance $\left(\mathrm{R}_{\mathrm{d}}\right)$ measurements of both the normal (black line) and cancer (red line) ex vivo breast sample over the whole spectrum range; (b) the diffuse reflectance ( $\mathrm{R}_{\mathrm{d}}$ ) measurements after applying normalization to the raw data to neglect the intensity effect and focus on the wavelength differentiation highlighting the three noticeable ranges $(630-680 \mathrm{~nm}),(720-770 \mathrm{~nm})$, and $(830-880 \mathrm{~nm})$.
Moreover, to validate the selected optimum wavelengths range from the signal of Fig. 4(b), we calculate the Standard Deviation $\left(\mathrm{S}_{\mathrm{d}}\right)$ between the normal and cancer tissue spectrum signature with spectral step $40 \mathrm{~nm}$, which shows that the highest contrast wavelength at $680 \mathrm{~nm}$, as presented in Table 1.

The Combination of the Measured $\left(\mathrm{R}_{\mathrm{d}}\right)$ Signals of the normal tissue and the tumor regions for the investigated Ten ex vivo breast samples were displayed in Fig. A4 in the Appendix. Moreover, the descriptive analysis of the average signals of the different investigated Ten ex vivo breast samples Fig. A4 to highlight the optimum wavelength to differentiate between the Normal tissue and the Tumor regions as illustrated in Table A2 in the appendix. Where Table 1 and Table A2 show that the highest contrast at the wavelength at $680 \mathrm{~nm}$ and the lowest contrast at wavelength $400 \mathrm{~nm}$.

To cover the band from 400 to $1000 \mathrm{~nm}$, we calculate 128 frames for each investigated sample with $4.68 \mathrm{~nm}$ resolution. However, it's difficult to process the 128 images, so to reduce the time of the image processing, we used the guidance data from the (1-D) signal at Fig. 4(b) and Fig. A4 in the Appendix beside the data from Table 1 and Table A2 in the Appendix. Furthermore, we examine the selection of the spectral image every $100 \mathrm{~nm}$ starting from $400 \mathrm{~nm}$ till $1000 \mathrm{~nm}$. Then, applying the custom algorithm (Normalization and Moving average filter $\mathrm{k}=10$ ) to remove the background noise and enhance the image processing, beside neglecting the intensity effect to focus for the wavelength differentiation between the normal tissue and malignant regions, as shown in Fig. 5. We exploited the histogram analysis to select the optimum spectral image rather than the visual selection, as displayed in Fig. 5(h), Fig. 5(i), and more clarified in Fig. A5 in the Appendix. 
Table 1 The measured diffuse reflectance ( $\left.R_{d}\right)$ of the ex vivo breast sample of the breast tissue to highlight the highest contrast between the normal and cancer tissue at the various spectrum range.

\begin{tabular}{|c|c|c|c|c|c|c|c|}
\hline Wavelength $(\mathbf{n m})$ & $\mathbf{4 0 0}$ & $\mathbf{4 4 0}$ & $\mathbf{4 8 0}$ & $\mathbf{5 2 0}$ & $\mathbf{5 6 0}$ & $\mathbf{6 0 0}$ & $\mathbf{6 4 0}$ \\
\hline Breast tissue reflection $\left(\mathrm{R}_{\mathrm{d}}\right)$ & 40.2 & 174.2 & 695.3 & 1777.9 & 2334.9 & 2420.5 & 1933.9 \\
\hline Cancer tissue reflection $\left(\mathrm{R}_{\mathrm{d}}\right)$ & 23.3 & 80.1 & 270.8 & 466.9 & 607.2 & 727.1 & 745.8 \\
\hline (Şd) after Normalization & 0.001 & 4.243 & 12.02 & 19.09 & 25.46 & 2.12 & 37.48 \\
\hline
\end{tabular}

\begin{tabular}{|c|c|c|c|c|c|c|c|}
\hline Wavelength $(\mathbf{n m})$ & $\mathbf{6 8 0}$ & $\mathbf{7 2 0}$ & $\mathbf{7 6 0}$ & $\mathbf{8 0 0}$ & $\mathbf{8 4 0}$ & $\mathbf{8 8 0}$ & $\mathbf{9 2 0}$ \\
\hline Breast tissue reflection $\left(\mathrm{R}_{\mathrm{d}}\right)$ & 1236.6 & 783.6 & 729.8 & 570.4 & 241.0 & 311.6 & 144.3 \\
\hline Cancer tissue reflection $\left(\mathrm{R}_{\mathrm{d}}\right)$ & 711.9 & 372.5 & 499.8 & 383.2 & 175.1 & 221.6 & 100.2 \\
\hline (Ş, $)$ after Normalization & 79.903 & 30.406 & 65.56 & 26.16 & 22.63 & 28.99 & 11.31 \\
\hline
\end{tabular}
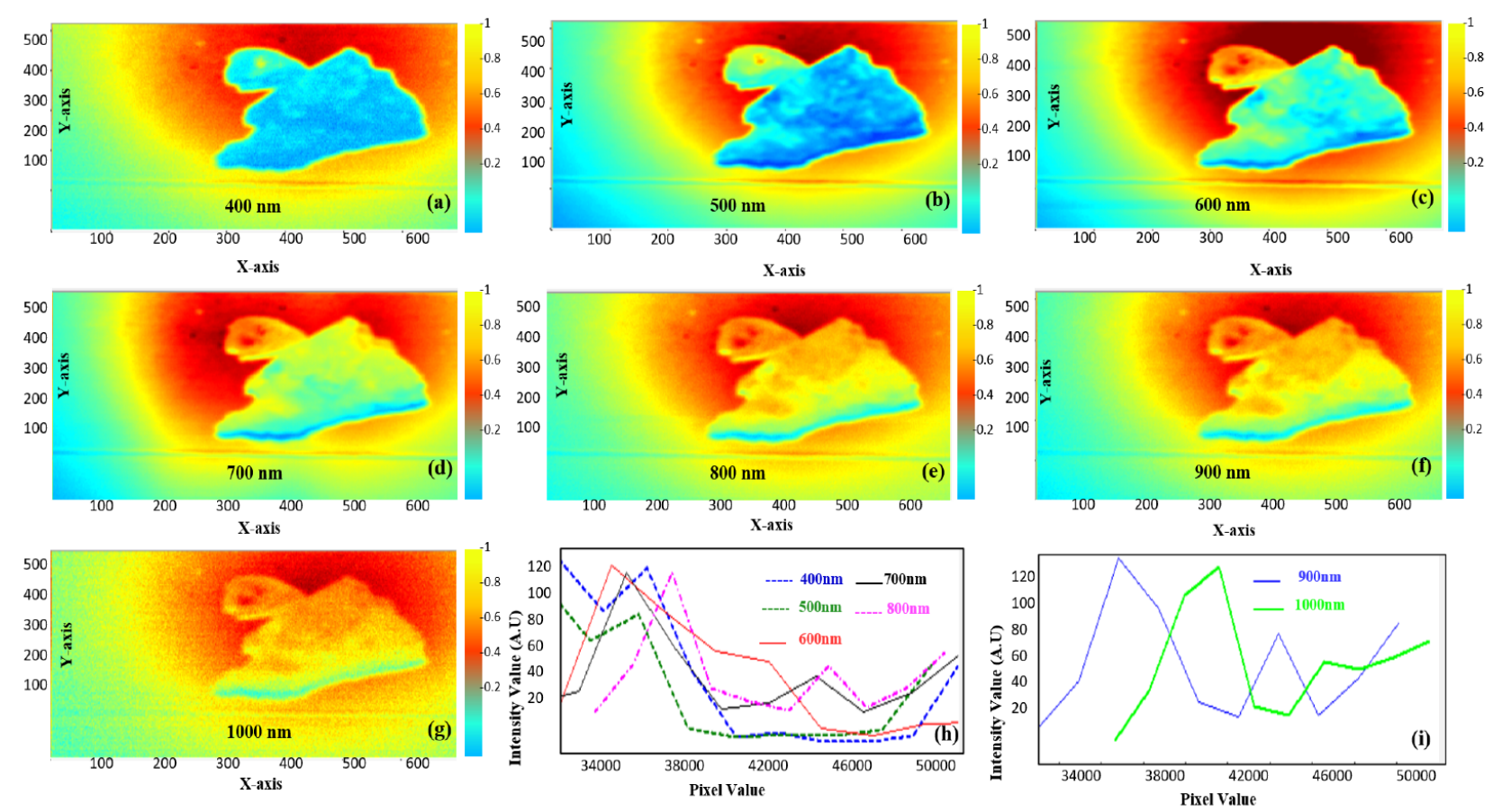

Fig. 5 The hyperspectral image of the investigated breast samples at wavelength range (400 1000 nm); (a) the captured ex vivo breast sample image at wavelength $400 \mathrm{~nm}$, (b) wavelength $500 \mathrm{~nm}$, (c) wavelength $600 \mathrm{~nm}$, (d) wavelength $700 \mathrm{~nm}$, (e) wavelength $800 \mathrm{~nm}$, (f) wavelength $900 \mathrm{~nm}$, (g) wavelength $1000 \mathrm{~nm}$, (h) comparing the image contrast at wavelengths $(400-800 \mathrm{~nm}$ ) by the histogram analysis to distinguish between the normal tissue and the malignant regions, (i) the histogram analysis at wavelengths $(900-1000 \mathrm{~nm})$.

From the histogram analysis, we could select the optimum spectral image with the highest contrast at the wavelength $(600 \mathrm{~nm})$ and the lowest contrast image $(400 \mathrm{~nm})$ to apply our custom subtraction algorithm to remove the DC background noise, image enhancement, then apply the $\mathrm{K}$-mean segmentation $(\mathrm{K}=8)$. Finally, the contour delineation of the variable threshold regions of the tumor regions in the breast sample, as illustrated in Fig. 6. For more clarification we apply the algorithm, on the whole, investigated samples are displayed in Fig. A6, Fig. A7, and Fig. A8 in the Appendix.

\section{Discussion}

There are an escalating interest in enlightening breast malignant identification methods that exploiting clinical imaging devices regarding its efficient therapeutic of breast tumors depending upon its proper and early detection [9]. Essentially, we could identify malignant early before the primary tumor metastasizes [5]. 

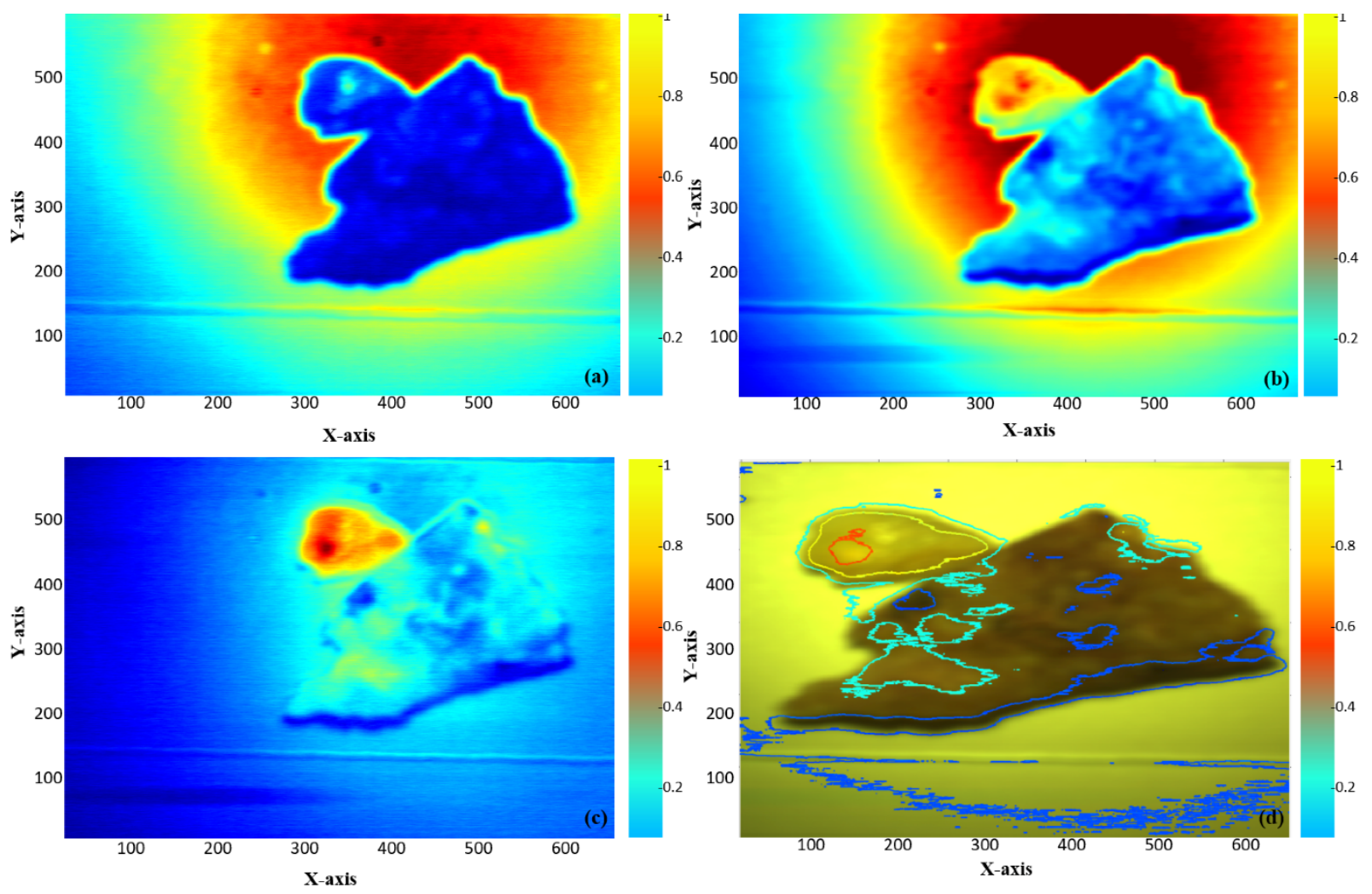

Fig. 6 The investigated ex vivo breast sample \#1 after applying the image processing, (a) the HS image of the breast sample at wavelength $400 \mathrm{~nm}$, (b) the HS image of the breast sample at wavelength at $600 \mathrm{~nm}$, (c) the spectral difference by subtraction of the HS image at wavelength 600 from the image at wavelength $400 \mathrm{~nm}$ to remove the DC background noise, (d) the contour delineation of the tumor variable threshold regions of in the breast sample.

Conventional methods for pathological investigations rely on the manual examination of morphological indicators of the samples by qualified pathologists. Where computer-aided diagnostic (CAD) systems could enhance the results and minimize the time of investigations [16]. Such innovations are planned to improve the diagnosis, make it reproducible and quantitative, and reduce time consumption in the assessment of samples [18].

During recent years, numerous investigations have been executed on the early recognition of malignant growth utilizing noninvasive methods in lieu of customary excisional biopsy. The HSI technology had been exploited in the detection of gastric tumors $[65,66]$, prostate cancer [67], an integrated endoscopic system with a hyperspectral camera to detect colorectal cancer [68], and breast cancer [45, 47].

We established an optical system setup exploiting a hyperspectral (HS) camera with a range $(400 \sim 100 \mathrm{~nm})$ with poly-chromatic source lights with wavelength $(380 \sim 1050 \mathrm{~nm})$, as shown in Fig. 2(a). Measuring the diffuse reflection $\left(\mathrm{R}_{\mathrm{d}}\right)$ of the ten-investigated ex vivo breast sample to select the optimum spectral image to differentiate between the normal and tumor in the near infra-red and visible (NIR-VIS) spectrum.

The raw data of the measured $\left(\mathrm{R}_{\mathrm{d}}\right)$ for the investigated ex vivo breast sample were displayed in Fig. 4(a), which emphasize the differentiation between the normal tissue and cancer region in range $(550 \sim 650 \mathrm{~nm})$. However, to avoid the variations of the intensity regarding the difference of the tissue optical properties we normalized the signals. To neglect the effect of the intensity and to evaluate the wavelength differentiation of the investigated samples, we normalized the measured initial data and focused on the spectral variations, as displayed in Fig. 4(b). We noticed that the wavelength range $(395-495 \mathrm{~nm})$ was almost identical and the variation between the normal and cancer was barely small. Additionally, there was noticeable variation in three ranges $(630-680 \mathrm{~nm}),(720-770 \mathrm{~nm})$, and $(830-880 \mathrm{~nm})$.

The experiment was repeated on the whole ten investigated ex vivo breast samples to measure the ( $\left.\mathrm{R}_{\mathrm{d}}\right)$ of the normal tissue as displayed in the worksheet in Fig. A2 in the Appendix. Although, the worksheet displayed only eight different signals (cannot display more than 9-windows at the same time) in Fig. A2(a) to Fig. A2(h) for sample \#1 to sample \#8, Consecutively. The magnified image for the combination (Rd) signals of the normal tissue for the investigated eight-samples regards the source light reference was shown in Fig. A2(i) in the Appendix. Furthermore, the measured $\left(\mathrm{R}_{\mathrm{d}}\right)$ of the tumor tissue was presented in Fig. A3 in the Appendix and the magnified image for the combination $\left(\mathrm{R}_{\mathrm{d}}\right)$ signals of the tumor regions for the investigated eight-samples regards the source light reference, as presented in Fig. A2(i) in the Appendix.

Moreover, to validate the selected optimum wavelength range from the measured signal of Fig. 4(b), 
we calculated the standard deviation $\left(\mathrm{S}_{\mathrm{d}}\right)$ between the normal and cancer tissue spectrum signature with spectral step $40 \mathrm{~nm}$, as presented in Table 1 . Furthermore, the combination of the measured $\left(\mathrm{R}_{\mathrm{d}}\right)$ signals regarding the normal tissue and the tumor regions for the investigated ten ex vivo breast samples were displayed in Fig. A4 in the Appendix. Additionally, we calculated the descriptive analysis of the average signals from Fig. A4 to highlight the optimum wavelength for differentiation between the normal tissue and the tumor regions as illustrated in Table A2. However, Table 1 and Table A2 show that the highest contrast wavelength for differentiation at $680 \mathrm{~nm}$ and the lowest contrast wavelength at $400 \mathrm{~nm}$.

Although to cover the band from 400 to $1000 \mathrm{~nm}$, we calculate 128 frames with $4.68 \mathrm{~nm}$ resolution. However, it is difficult and time-consuming to process the 128 images, so to reduce the time of the image processing, we used the guidance data from the (1-D) signal at Fig. 4(b) and Fig. A4 in the Appendix beside the data from Table 1 and Table A2 in the Appendix leading to the selection of the spectral image every $100 \mathrm{~nm}$ step, starting from $400 \mathrm{~nm}$ till $1000 \mathrm{~nm}$, as noticed from the investigation that $100 \mathrm{~nm}$ as a step in the illustrating signal was enough, so we can choose the closest result wavelength, as more clarified in Fig. 5.

After applying the custom algorithm (normalization and moving average filter $\mathrm{k}=10$ ) to remove the background noise and enhance the image processing, besides neglecting the intensity effect on the selected spectral images $(400,500,600,700,800,900,1000 \mathrm{~nm})$. We noticed visually from Fig. 5 , that spectral images at wavelength $600 \mathrm{~nm}$ and $700 \mathrm{~nm}$ were in higher contrast to identifying the tumor region. However, for more validation, we exploited the histogram analysis to distinguish between the normal tissue and the malignant regions, as shown in Fig. 5(h) and Fig. 5(i) and more illustrated in Fig. A5 in the Manuscript Appendix. Where, from the histogram analysis it identifies that the spectral image at $600 \mathrm{~nm}$ was higher in contrast and with minimum noise in the image processing, which provides us with better information to differentiate between normal tissue and tumor regions.

Although after selecting the optimum spectral image from the histogram analysis, we apply the image processing to improve the image contrast. Where, the highest contrast image at a wavelength $(600 \mathrm{~nm})$, as shown in Fig. 6(a), and the lowest contrast image at a wavelength $(400 \mathrm{~nm})$, as shown in Fig. 6(b). Next, applying the custom subtraction algorithm to remove the DC background noise, as display in Fig. 6(c). Furthermore, applying K-mean segmentation $(\mathrm{K}=8)$, and contour delineation for the variable threshold regions of the breast tumor regions, as illustrated in Fig. 6 (d).

Finally, repeating the same previous process on the whole various investigated samples to validate the algorithm reliability and ensure its reproducibility for the given features between the various samples of the same class, as displayed in Fig. A6, Fig. A7, and Fig. A8 in the Appendix.

Although, the worksheets of the investigated sample \#2, sample \#3, and sample \#4 identified the two high contrast images at wavelengths $(600 \mathrm{~nm}$ and $700 \mathrm{~nm}$ ) with respect to the lowest contrast image at wavelength $400 \mathrm{~nm}$. However, after enhancing the contrast of the spectral image we noticed that the optimum image in differentiation between the normal tissue and the malignant regions was more identified at wavelength $600 \mathrm{~nm}$, as shown in Fig. A6(e), Fig. A7(e), and Fig. A8(e) in the Appendix. Next, applying the subtraction algorithm on the investigated samples to highlight the contour mapping of the variable threshold regions of the tumors, as displayed in Fig. A6(i), Fig. A7(i), and Fig. A8(i) in the Appendix.

The output result of the custom algorithm on the investigated samples was validated by comparing its results with respect to the pathological report. Where, the investigated RGB image of the ex vivo breast sample \#1 and sample \#3 were displayed in Fig. A9 (a) and Fig. A10(a), respectively in the Appendix. The result of the applied custom algorithm is shown in Fig. A (b) and Fig. A10(b) in the Appendix.

\section{Conclusion}

The presented approach reveals promising results regards the diagnostic applications of breast cancer to assist the pathologist and in the real-time investigations for assisting the surgeons. Where experiment results clarify that the optimum wavelength at $680 \mathrm{~nm}$ for diagnostic applications and the ideal spectral image at $600 \mathrm{~nm}$ to discriminate between the normal and malignant tissue. In Future work, we going to update the system hardware like the HS camera and expanding the source light wavelength range. Moreover, we could utilize a $\mathrm{Q}$ - switched pulsed laser with a custom optical system $(686.9 \mathrm{~nm})$ for breast tumor ablation without normal tissue damage.

\section{Disclosures}

The authors stated and declare that No funders for this study. The authors stated and declare that No conflict or competing of interests. All experimental and investigation trails was approved and validated from "Ain Shams University" - Medical College - Ethics Committee. No experimental investigation was performed on individuals within this research study. All the co-authors are agreed for the research study publication. The authors stated and declare that all data is exist and available. The authors stated and declare that all the source codes are available. 


\section{References}

1. C. Review, "Global, Regional, and National Cancer Incidence, Mortality, Years of Life Lost, Years Lived With Disability, and Disability- Adjusted Life-years for 32 Cancer Groups, 1990 to 2015 A Systematic Analysis for the Global Burden of Disease Study," JAMA Oncology 3(4), 524-548 (2017).

2. F. Bray, J. Ferlay, I. Soerjomataram, R. L. Siegel, L. A. Torre, and A. Jemal, "Global cancer statistics 2018: GLOBOCAN estimates of incidence and mortality worldwide for 36 cancers in 185 countries," CA: A Cancer Journal for Clinicians 68(6) 394-424 (2018).

3. R. L. Siegel, K. D. Miller, and A. Jemal, “Cancer statistics, 2019,” CA: A Cancer Journal for Clinicians 69(1), 7-34 (2019).

4. K. Saika, T. Sobue, "Cancer statistics in the world," Gan To Kagaku Ryoho 40, 2475-2480 (2013).

5. R. Siegel, D. Naishadham, and A. Jemal, "Cancer statistics, 2013," CA: A Cancer Journal for Clinicians 63, 11-30 (2013).

6. J. Shan, H.D. Cheng, J. Shan, W. Ju, Y. Guo, and L. Zhang, “Automated breast cancer detection and classification using ultrasound images : A survey Automated breast cancer detection and classification using ultrasound images : A survey," Pattern Recognition 43(1), 299-317 (2010).

7. D. Saravanan, D. Joseph, and S. Vaithyasubramanian, "Effective utilization of image information using data mining technique," in Recent Trends and Advances in Artificial Intelligence and Internet of Things, V. Balas, R. Kumar, R. Srivastava (eds.), Springer, Cham, 207-215 (2020).

8. S. Alrahbi, P. M. Y. Chan, B. C. S. Ho, M. D. W. Seah, J. J. C. Chen, and E. Y. Tan, "Extent of margin involvement, lymphovascular invasion, and extensive intraductal component predict for residual disease after wide local excision for breast cancer," Clinical Breast Cancer 15, 219-226 (2015).

9. A. L. Merrill, S. B. Coopey, R. Tang, M. P. McEvoy, M. C. Specht, K. S. Hughes, M. A. Gadd, and B. L. Smith, "Implications of New Lumpectomy Margin Guidelines for Breast-Conserving Surgery: Changes in Reexcision Rates and Predicted Rates of Residual Tumor," Annals of Surgical Oncology 23, 729-734 (2016).

10. S. Alrahbi, P. M. Y. Chan, B. C. S. Ho, M. D. W. Seah, J. J. C. Chen, and E. Y. Tan, "Extent of margin involvement, lymphovascular invasion, and extensive intraductal component predict for residual disease after wide local excision for breast cancer," Clinical Breast Cancer 15(3), 219-226 (2015).

11. E. Kho, B. Dashtbozorg, L. L. de Boer, K. K. Van de Vijver, H. J. C. M. Sterenborg, and T. J. M. Ruers, "Broadband hyperspectral imaging for breast tumor detection using spectral and spatial information," Biomedical Optics Express 10(9), 4496 (2019).

12. R. G. Pleijhuis, M. Graafland, J. De Vries, J. Bart, J. S. De Jong, and G. M. Van Dam, “Obtaining Adequate Surgical Margins in Breast-Conserving Therapy for Patients with Early-Stage Breast Cancer: Current Modalities and Future Directions," Annals of Surgical Oncology 16, 2717-2730 (2009).

13. E. L. Vos, A. Jager, C. Verhoef, A. C. Voogd, and L. B. Koppert, "Overall survival in patients with a re-excision following breast conserving surgery compared to those without in a large population-based cohort," European Journal of Cancer 51(3), 282-291 (2015).

14. C. D. Runowicz, C. R. Leach, N. L. Henry, K. S. Henry, H. T. Mackey, R. L. Cowens-Alvarado, R. S. Cannady, M. L. Pratt-Chapman, S. B. Edge, and L. A. Jacobs, "American cancer society/American society of clinical oncology breast cancer survivorship care guideline," CA: A Cancer Journal for Clinicians 66(1), 43-73 (2016).

15. S. L. Van Es, "Digital pathology: semper ad meliora," Pathology 51(1), 1-10 (2019).

16. A. Madabhushi, G. Lee, "Image analysis and machine learning in digital pathology: Challenges and opportunities," Medical Image Analysis 33, 170-175 (2016).

17. T. J. Flotte, D. A. Bell, “Anatomical pathology is at a crossroads," Pathology 50(4), 373-374 (2018).

18. J. J. Keating, C. Fisher, R. Batiste, and S. Singhal, "Advances in intraoperative margin assessment for breast cancer," Current Surgery Reports 4(4), 15 (2016).

19. K. Butler-Henderson, A. H. Lee, R. I. Price, and K. Waring, "Intraoperative assessment of margins in breast conserving therapy: A systematic review," Breast 23(2), 112-119 (2014).

20. E. R. St John, R. Al-Khudairi, H. Ashrafian, T. Athanasiou, Z. Takats, D. J. Hadjiminas, A. Darzi, and D. R. Leff, "Diagnostic accuracy of intraoperative techniques for margin assessment in breast cancer surgery," Annals of surgery 265(2), 300-310 (2017).

21. D. P. A. Versteegden, L. G. G. Keizer, L. E. M. Duijm, C. A. P. Wauters, and L. J. A. Strobbe, "Performance characteristics of specimen radiography for margin assessment for ductal carcinoma in situ : a systematic review," Breast Cancer Research and Treatment 166, 669-679 (2017).

22. K. Esbona, Z. Li, and L. G. Wilke, "Intraoperative imprint cytology and frozen section pathology for margin assessment in breast conservation surgery: a systematic review," Annals of surgical oncology 19(10), 3236-3245 (2012). 
23. F. K. Althoubaity, A. Merdad, N. Y. Akeel, N. H. Zaidi, and A. O. Sultan, "Frozen Section Analysis of Breast Lumpectomy Margins," Surgical Science 8(6), 269-277 (2017).

24. I. Journal, O. Medical, A. Yilmaz, M. Bayrak, K. Ozerkan, and H. Ozan, “Accuracy of Intraoperative Frozen Section Analysis in Borderline Ovarian Tumor and the Factors Affecting It," International Journal of Medical Science and Clinical Invention 7(9), 7-12 (2020).

25. H. J. C. M. Sterenborg, E. Kho, L. L. de Boer, K. K. van de Vijver, and T. M. Ruers, "Hyperspectral imaging for intraoperative margin assessment during breast cancer surgery," in Asia Communications and Photonics Conference, AF1K-6 (2016).

26. A. Sahu, Hyperspectral Imaging to Discern Malignant and Benign Canine Mammary Tumors, Master Thesis, Temple University, Philadelphia, USA (2012).

27. L. Ma, G. Lu, D. Wang, X. Qin, Z. G. Chen, and B. Fei, “Adaptive deep learning for head and neck cancer detection using hyperspectral imaging," Visual Computing for Industry, Biomedicine, and Art 2(1), 18 (2019).

28. M. H. Fouad Aref, A. A. R. Sharawi, and Y. H. El-Sharkawy, "Delineation of the Arm Blood Vessels Utilizing Hyperspectral Imaging to Assist with Phlebotomy for Exploiting the Cutaneous Tissue Oxygen Concentration," Photodiagnosis and Photodynamic Therapy 33, 102190 (2021).

29. H. Zhao, R. H. Webb, and B. Ortel, “A new approach for noninvasive skin blood imaging in microcirculation,” Optics \& Laser Technology 34(1), 51-54 (2002).

30. U. Rubins, A. Grabovskis, J. Cimurs, Z. Marcinkevics, and A. Caica, "Hyperspectral evaluation of skin blood oxygen saturation at baseline and during arterial occlusion," Proceedings of SPIE 10685, 106851A (2018).

31. T. Delineation, H. Fabelo, S. Ortega, R. L. Id, D. M. Id, G. M. Callic, E. Ju, D. Bulters, H. B. Id, A. Szolna, J. F. Piñeiro, C. Sosa, A. J. O. Shanahan, S. Bisshopp, D. Ravi, B. R. Kiran, A. Vega, B. Abelardo, G. Yang, and B. Stanciulescu, “An Intraoperative Visualization System Using Hyperspectral Imaging to Aid in Brain,” Sensors 18(2), 430 (2018).

32. M. H. Aref, I. H. Aboughaleb, and Y. H. El-Sharkawy, "Tissue characterization utilizing hyperspectral imaging for liver thermal ablation," Photodiagnosis and Photodynamic Therapy 31, 101899 (2020).

33. N. Yavari, Optical spectroscopy for tissue diagnostics and treatment control, Doctoral Thesis, University of Bergen, Norway (2006).

34. J. R. Mourant, J. P. Freyer, A. H. Hielscher, A. A. Eick, D. Shen, and T. M. Johnson, "Mechanisms of light scattering from biological cells relevant to noninvasive optical-tissue diagnostics," Applied Optics 37(16), 3586-3593 (1998).

35. M. Li, Developing a technique for combining light and ultrasound for deep tissue imaging, Master Thesis, Lund University, Sweden (2018).

36. V. Tuchin, "Tissue Optics and Photonics: Light-Tissue Interaction II," Journal of Biomedical Photonics \& Engineering 2(3), 030201 (2016).

37. K. G. Wakim, "Physiology of the liver," The American Journal of Medicine 16(2), 256-271 (1954).

38. M. H. Aref, "Biomedical Research and Clinical Reviews," Biomedical Research and Clinical Reviews 1, 1-13 (2020).

39. S. Swierczynski, F. Primavesi, E. Klieser, T. Kiesslich, T. Jäger, R. Illig, D. Neureiter, D. Ofner, and S. Stättner, "382. Thermographic monitoring of radiofrequency and microwave ablation in a perfused porcine liver model," European Journal of Surgical Oncology 40(11), S147 (2014).

40. M. De Landro, M. Barberio, E. Felli, V. Agnus, M. Pizzicannella, M. Diana, and P. Saccomandi, "Hyperspectral image-based analysis of thermal damage in living liver undergoing laser ablation," Proceedings of SPIE 11362 , 113620G (2020).

41. R. Sucher, A. Athanasios, H. Köhler, T. Wagner, M. Brunotte, A. Lederer, I. Gockel, and D. Seehofer, "Hyperspectral Imaging (HSI) in anatomic left liver resection," International Journal of Surgery Case Reports 62, 108-111 (2019).

42. M. De Landro, P. Saccomandi, M. Barberio, E. Schena, S. Member, and M. Diana, "Hyperspectral imaging for thermal effect monitoring in in vivo liver during laser ablation Hyperspectral imaging for thermal effect monitoring in in vivo liver during laser ablation," In 2019 41st Annual International Conference of the IEEE Engineering in Medicine and Biology Society, 1851-1854 (2019).

43. M. A. Calin, S. V. Parasca, D. Savastru, and D. Manea, "Hyperspectral imaging in the medical field: present and future," Applied Spectroscopy Reviews 49(6), 435-447 (2014).

44. M. Aref, I. H. Aboughaleb, A.-B. Youssef, and Y. El-Sharkawy, "Novel Approach Exploiting the Hyperspectral Imaging System for Breast Cancer Therapy and Diagnosis," Archives of Breast Cancer 7(4), 189-201 (2020).

45. I. H. Aboughaleb, M. H. Aref, and Y. H. El-Sharkawy, "Photodiagnosis and Photodynamic Therapy Hyperspectral imaging for diagnosis and detection of ex-vivo breast cancer," Photodiagnosis and Photodynamic Therapy 31 , 101922 (2020).

46. M. H. Aref, I. H. Aboughaleb, and Y. H. El-Sharkawy, "Custom optical imaging system for ex-vivo breast cancer detection based on spectral signature," Surgical Oncology 35, 547-555 (2020).

47. T. D. O. Sullivan, A. E. Cerussi, D. J. Cuccia, B. J. Tromberg, T. D. O. Sullivan, A. E. Cerussi, D. J. Cuccia, and B. J. Tromberg, "Diffuse optical imaging using spatially and temporally modulated light Diffuse optical imaging using spatially and temporally," Journal of Biomedical Optics 17(7), 071311 (2012). 
48. F. Baum, "1 Development, Anatomy, and Physiology of the Mammary Gland,” Breast Cancer: Diagnostic Imaging and Therapeutic Guidance, 1-3 (2017).

49. H. Ellis, V. Mahadevan, “Anatomy and physiology of the breast,” Surgery (Oxford) 31(1), 11-14 (2013).

50. S. Pandya, R. G. Moore, "Breast development and anatomy," Clinical obstetrics and gynecology 54(1), 91-95(2011).

51. G. Mostafa, L. Cathey, and F. L. Greene (Eds.), Breast Anatomy, in Review of Surgery, Springer, New York, 161162 (2006).

52. C. Frantz, K. M. Stewart, and V. M. Weaver, "The extracellular matrix at a glance,” Journal of Cell Science 123(24) 4195-4200 (2010).

53. T. R. Cox, J. T. Erler, "Remodeling and homeostasis of the extracellular matrix: Implications for fibrotic diseases and cancer," Disease Models and Mechanisms 4(2), 165-178 (2011).

54. R. G. Aswathy, Y. Yoshida, "Near-infrared quantum dots for deep tissue imaging," Analytical and Bioanalytical Chemistry 397, 1417-1435 (2010).

55. C. Bremer, "Fluorescence imaging with near-infrared light: new technological advances that enable in vivo molecular imaging," European Radiology 13, 195-208 (2003).

56. Q. Miao, K. Pu, “Organic Semiconducting Agents for Deep-Tissue Molecular Imaging: Second Near-Infrared Fluorescence,” Advanced Materials 30(49), 1801778 (2018).

57. R. J. DiPerna, P. L. Lions, "Ordinary differential equations, transport theory and Sobolev spaces," Inventiones mathematicae 98, 511-547 (1989).

58. K. Rinzema, L. H. P. Murrer, and W. M. Star, "Direct experimental verification of light transport theory in an optical phantom," Journal of the Optical Society of America A 15(8), 2078-2088 (1998).

59. S. S. M. Noor, K. Michael, S. Marshall, and J. Ren, "Hyperspectral image enhancement and mixture deep-learning classification of corneal epithelium injuries," Sensors 17(11), 2644 (2017).

60. V. Kumar, P. Gupta, "Importance of Statistical Measures in Digital Image Processing," International Journal of Emerging Technology and Advanced Engineering 2(8), 56-62 (2012).

61. S. Engineering, J. Zhang, and J. Hu, "Image Segmentation Based on 2D Otsu Method with Histogram Analysis," In 2008 International Conference on Computer Science and Software Engineering 6, 105-108 (2008).

62. M. D. Abràmoff, P. J. Magalhães, and S. J. Ram, “Image processing with ImageJ," Biophotonics International 11(7), 36-42 (2004).

63. O. Holub, S. T. Ferreira, "Quantitative histogram analysis of images," Computer Physics Communications 175(9), $620-623$ (2006).

64. H. Akbari, K. Uto, Y. Kosugi, K. Kojima, and N. Tanaka, "Cancer detection using infrared hyperspectral imaging," Cancer Science 102(4), 852-857 (2011).

65. S. Kiyotoki, J. Nishikawa, T. Okamoto, K. Hamabe, M. Saito, A. Goto, Y. Fujita, Y. Hamamoto, Y. Takeuchi, S. Satori, and I. Sakaida, "New method for detection of gastric cancer by hyperspectral imaging: a pilot study," Journal of Biomedical Optics 18(2), 026010 (2013).

66. M. Nathan, A. S. Kabatznik, and A. Mahmood, "Hyperspectral imaging for cancer detection and classification," In 2018 3rd Biennial South African Biomedical Engineering Conference, 1-4 (2018).

67. R. Kumashiro, K. Konishi, T. Chiba, T. Akahoshi, S. Nakamura, M. Murata, M. Tomikawa, T. Matsumoto, Y. Maehara, and M. Hashizume, "Integrated endoscopic system based on optical imaging and hyperspectral data analysis for colorectal cancer detection," Anticancer Research 36(8), 3925-3932 (2016).

68. I. H. Aboughaleb, M. H. Aref, and Y. H. El-Sharkawy, "Hyperspectral imaging for diagnosis and detection of exvivo breast cancer," Photodiagnosis and Photodynamic Therapy 31, 101922 (2020). 